\title{
Lemons D. S.: A Student's Guide to Dimensional Analysis
}

\author{
Cambridge University Press, Cambridge, 2017. ISBN 978-1-316-61381-8.
}

ANDRZEJ ICHA ${ }^{1}$

"The Dimensional Analysis, called also the Principle of Similitude, is a computing method used in practical problems of physics, technics, natural philosophy and other disciplines" (Drobot S., On the Foundations of Dimensional Analysis. Studia Mathematica (1953), vol. 14. 1, 84-99). At the core of dimensional analysis (DA) is the concept of similarity. In physical terms, this notion refers to some equivalence between two processes or phenomena that are actually different. The main result of a DA of a problem is the Buckingham's $\Pi$ theorem which reduces the number of variables in the problem and establishes the existence of dimensionless representation of physical relations.

This book introduces the reader to the captivating world of DA through an adequate number of examples and just as many basics which are necessary to understand the working principles and foundations of the method. The book consists of seven chapters and one appendix.

Chapter 1, Introduction, motivates the use of DA and discusses its basic concepts. The fundamental ideas are introduced and explained, namely, the principle of dimensional homogeneity, and the symmetry principle, as the projections of the following axiom: physical laws do not depend upon arbitrariness in the choice of basic units of measurements, that is, the physical laws are homogeneous. So, the requirement of dimensional homogeneity imposes restrictions on the quantities involved in a physical problem, namely, the dimension function is always in

1 Pomeranian Academy in Slupsk, Institute of Mathematics, ul. Kozietulskiego 6-7, 76-200 Słupsk, Poland. E-mail: majorana38@gmail.com the form of a power-law monomial. As a consequence, two existing approaches are described and elucidated: the Rayleigh's Indicial algorithm, and the Buckingham's $\Pi$ theorem. Two examples are presented to provide a better understanding of how it works: an ideal gas, in which the pressure depends on parameters describing its state, and the spring-mass system, together with the analysis of the possible mistakes in the art of modeling.

Chapters 2-7 provide many illustrative examples on how to use DA to investigate the structure of solutions for physical problems in various areas. The essence of the method is presented; for example, from mechanics, hydrodynamics, thermodynamics, electrodynamics, plasma physics, and quantum physics. The world of the fundamental dimensional constants is also considered.

Chapter 2, Mechanics, applies DA to the bestknown examples from a basic course in classic mechanics. First, the choice of length, mass, and time as fundamental dimensional quantities in mechanics is briefly justified. A few examples to clarify the DA methods concern the following issues: gas enclosed within a container; hanging spring-mass system; hanging, stretched cable, Helmholtz resonator, and two-body problem. Chapter 3, Hydrodynamics then continues the use of DA to typical problems in fluid mechanics. At the beginning, the analysis of governing parameters, involved in the fluid-flow description, is performed. Next, the water waves phenomena are discussed, including the small-amplitude gravity waves, and the capillary waves. Also, there is discussion of volume of the largest water drop, the terminal speed of an object falling in a viscous fluid, Stokes' law, hydraulic jump, as well as the equilibrium and non-equilibrium flow in pipes. 
Chapter 4, Temperature and Heat, deals with the process of heat transfer in natural media (solids, liquids, gases, etc.). In the general case these processes are described by a system of coupled non-linear partial differential equations that include the continuity, momentum, energy, and admixture's conservation equations. Therefore, a qualitative analysis of such complex phenomena may be significantly simplified by applying the DA. The results present in this chapter explain the physical essence of conduction, convection, and diffusion, and demonstrate the applications of DA to growth of ice thickness, stack effect, and to estimate turkey cooking time!

Chapter 5, Electrodynamics and Plasma Physics, contains a concise presentation of classical electrodynamics theory, together with the plasma concept, often called the fourth state of matter. It begins with a very brief review of the Maxwell's equations. The following DA examples are related to electrodynamics: the frequency of oscillations of a compass needle, the power of radiation from an accelerating charge, and the space charge limited current density (the Child-Langmuir law). Plasma is a special kind of ionized gas and usually consists of positively charged ions, electrons, and neutrals. The DA examples include: frequency of plasma oscillations, and the Pinch effect (the radial constriction of the plasma column).

The starting point of the quantum theory is associated with 1900, the year Max Planck gave a formula for the distribution of energy in the radiation from a black-body. Chapter 6, Quantum Physics, presents several DA examples, mainly, from the old quantum theory. The fundamental importance of Planck's constant is underlined first. Next, the Stefan-Boltzmann law and the Planck's law for black-body radiation are derived using the DA. Subsequently, the dimensional machinery to analyze properties of Bohr's model of the hydrogen atom is applied together with the discussion of atomic scale units. The simplest model of a quantum fluid provided by the ideal gas of particles subjected to the Fermi-Dirac statistics as well as the Bose-Einstein condensate is also described briefly. Finally, the quantum correction to the power of radiation from an accelerating charge is demonstrated and the dimensionless product called the fine structure constant is introduced.

Chapter 7, Dimensional Cosmology, offers a very terse introduction to "constantology", the discipline of physics which refers to the study of fundamental constants. Classical physics acquainted us with the Newtonian gravitational constant, the Boltzmann constant, the vacuum permittivity, and the speed of light. Quantum physics introduced another three fundamental constants, that is, the Planck's constant, the electron charge, and the electron mass. The "constantology" is illustrated with the following standard examples: the Eddington-Dirac ratio, the gravitational-electrostatic mass, and the classical electron radius. Two important issues, namely, the fine structure constant, and the Planck units are discussed in more detail. Also, 22 unique quantities, which can be interpreted in terms of particular kinds of fundamental interactions, quantum-mechanical effects, and certain particle properties, are listed.

Additionally, the book contains almost 40 problems regarding the dimensional analysis grouped at the end of each chapter. The problems are formulated in such a way that they form a good basis for the understanding of the role of dimensional analysis in scientific practice. The solutions to these problems are summarized in Appendix. It should be noted; however, that dimensional analysis is not an all-powerful discipline; often, the effective use of DA requires almost no understanding of the physical processes, and it applies usually to problems with a small number of input parameters, or when a poorly understood physical phenomenon is studied. In addition, it must be emphasized that the Rayleigh's method has limitations because of the assumption that an exponential relationship exists between the variables. So, today, the Buckingham's $\Pi$ theorem is strongly recommended.

In sum, the book is rather elementary. A background in undergraduate mathematics and physics, in particular linear algebra, basic calculus, and physics fundamentals, is sufficient as a prerequisite for the use of this book. It should, therefore, be accessible to a wide spectrum of students. For the graduate students of physics, geophysics, astrophysics, and 
engineering, as well as for research scientists and engineers in relevant fields, I additionally recommend The Pi-Theorem Applications to Fluid Mechanics and Heat and Mass Transfer, by L.P. Yarin (Springer, 2012) and Astrophysics, Clocks and Fundamental Constants (S. G. Karshenboim and E. Peik (Eds.), Springer, 2004).
Open Access This article is distributed under the terms of the Creative Commons Attribution 4.0 International License (http://creativecommons.org/licenses/by/4.0/), which permits unrestricted use, distribution, and reproduction in any medium, provided you give appropriate credit to the original author(s) and the source, provide a link to the Creative Commons license, and indicate if changes were made. 